\title{
Destructive effects of therapeutic ultrasound and gold nanoparticles on a breast carcinoma tumor model in BALB/c mice
}

\author{
A. Shanei ${ }^{*}$, M. Mirzaeiyan², H. Hejazi3 \\ ${ }^{1}$ Department of Medical Physics, School of Medicine, Isfahan University of Medical Sciences, Isfahan, Iran \\ ${ }^{2}$ Department of Medical Physics, School of Medicine, Isfahan University of Medical Sciences, Isfahan, Iran \\ ${ }^{3}$ Department of Parasitology and Mycology, School of Medicine, Isfahan University of Medical Sciences, Isfahan, \\ Iran
}

\section{- Original article}

\author{
*Corresponding authors: \\ Ahmad Shanei, Ph.D., \\ E-mail: \\ Shanei@med.mui.ac.ir \\ Revised: October 2019 \\ Accepted: November 2019 \\ Int. J. Radiat. Res., October 2020; \\ 18(4): 743-751 \\ DOI: 10.18869 /acadpub.ijrr.18.4.743
}

\begin{abstract}
Background: Acoustic cavitation which occurs at high intensities of ultrasound waves can be fatal for tumor cells; however, it can be used to destroy cancer cells as an efficient therapeutic method. On the other hand, it is known that the existence of nanoparticles in a liquid decreases the acoustic cavitation onset threshold. Materials and Methods: In this work, the combined effects of therapeutic ultrasound and gold nanoparticles (GNPs) on a breast carcinoma tumour model in BALB/c mice were studied. The tumour-bearing mice were divided into 4 groups (1) Control, (2) GNPs, (3) Ultrasound alone and (4) Ultrasound in the presence of GNPs. In groups 2 and 4, GNPs were injected into tumours. Therapeutic effects on tumours were evaluated by measuring relative tumour volume (RTV), doubling time $\left(T_{2}\right)$ and 5 -folding time $\left(T_{5}\right)$ for tumours volume. Results: The $T_{5}$ showed a significant difference between ultrasound in the presence of GNPs group and the other groups. The maximum $T_{2}$ and $T_{5}$ were found in the ultrasound in the presence of GNPs group. Conclusion: Combined effects of ultrasound and GNPs can be used as a method for increasing the therapeutic efficiency on tumor cells.
\end{abstract}

Keywords: Acoustic cavitation, gold nanoparticle, breast tumor model, relative tumor volume.

\section{INTRODUCTION}

In recent years, different methods of tumour treatment by ultrasound waves have been successfully developed (1). Applications of therapeutic ultrasound are based on its interactions with tissues, which create biological effects.

For hyperthermia induction, High intensity focused ultrasound (HIFU) is utilized. HIFU, produced by focalized transducers, can be applied either as the demolition of the tumour cells or as palliative therapy. It has been utilized to treat solid tumours and investigators have confirmed its efficacy (2).

Alternatively, the biological effects and therapeutic applications of the low intensity ultrasound (LIU) on tumour cells are under investigation. There is evidence indicating that the responses of tumour cells to LIU are more severe than healthy cells, in other words tumour cells are more sensitive to LIU than the healthy ones. In the recent years, the therapeutic applications of LIU have generated an expanding field (3). Researchers have look into improving treatment methods of tumors, in the meantime reducing their side effects (4). Enhanced lethality of anticancer agents with ultrasound exposure has made it possible to apply a lower drug dosage and at the same time increase the patient's tolerance to chemotherapy (4). Moreover, ultrasound waves have an important role in the transfer of therapeutic agents into the target tissue (5). 
Biological effects of ultrasound are mainly caused by heat, mechanical effects and cavitation. Among the mentioned effects, cavitation is the most important one (6). Acoustic cavitation in a liquid could occur in 2 modes of stable and inertial, when it is irradiated with ultrasound high intensities (7). In stable cavitation, bubbles can oscillate around an equilibrium radius without collapsing. While in inertial mod, bubbles expand up 2-3 times their resonant size and finally collapse in a compression half-cycle (8).

It has been experimentally shown that the collapsing cavity produces high temperatures (up to $5000^{\circ} \mathrm{K}$ ) in tissue and increases the pressure (up to $1800 \mathrm{~atm}$ ) for an extremely short period in a micron dimensions (8).

Furthermore, cavitation collapse produces free radicals from the water breakdown and consequently generates the hydrogen and hydroxyl radicals, as well as other agents such as hydrogen peroxide, singlet oxygen and superoxide ions ${ }^{(9)}$.

The inertial cavitation can be utilized to destroy cancerous cells as an efficient therapeutic method (8).

The use of high-intensity ultrasound is one of the existing challenges in ultrasound therapy. The treatment by the ultrasound depends on the cavitation process; therefore, high intensity ultrasound is an important necessity. Beside, high intensity ultrasound can induce side effects on the healthy tissues that have surrounded the tumor.

On the other hand, the existence of nanoparticles in a liquid presents a nucleation site for the cavitation bubble which dramatically decreases the cavitation onset threshold. This decrease in the cavitation onset threshold leads to an increase in the number of bubbles as a liquid is irradiated by the ultrasound (10).

Thus, in this context, one approach is based on providing the nucleation sites that participate in the formation of cavities to reduce the threshold intensity needed for cavitation.

On the other hand, gold nanoparticles (GNPs) are novel nano-materials that have the potential to be used in cancer treatment mainly due to their exceptional optical properties (11). In the meantime, low toxicity and good uptake of GNPs by cells, have made GNPs attractive for therapeutic applications (12).

To be more concise, the ultrasound waves' role in the presence of GNPs in creating and increasing cavitation as well as its anti-tumor effect on breast carcinoma tumor in Balb/c mice was investigated in this research.

\section{MATERIALS AND METHODS}

\section{Synthesis of GNPs}

All the chemical substances and reagents used in this study were purchased from Merck, Germany and Sigma Aldrich, USA. Hydrogen tetrachloroaurate (III), trihydrate and sodium citrate dihydrate $\left(\mathrm{C}_{6} \mathrm{H}_{5} \mathrm{Na}_{3} \mathrm{O}_{7} .2 \mathrm{H}_{2} \mathrm{O}\right)$ were purchased from Merck.

GNPs were prepared by the chemical reduction of $\mathrm{HAuCl}_{4}$ in the presence of citrate (13). For this step, $50 \mathrm{~mL}$ of $0.01 \% \mathrm{HAuCl}_{4}$ solution was heated to boiling temperature while stirring in a $100 \mathrm{~mL}$ round bottom flask. Then, a few hundred $\mu \mathrm{L}$ of $1 \%$ of trisodium citrate solution was quickly added to $\mathrm{HAuCl}_{4}$ one. The solution colour was changed within several minutes from yellow to red or purple. This depended on the size of the GNPs; the amount of citrate solution determines the size of the GNPs. The $350 \mu \mathrm{L}$ of $1 \%$ trisodium citrate solution obtains GNPs with around $23 \mathrm{~nm}$ in diameter.

\section{Characterization techniques}

UV-visible (UV-vis) absorption spectroscopic measurements were recorded on a UV-vis spectrometer (Agilent Cary 100), using quartz cells of $1 \mathrm{~cm}$ path length and water as the reference solvent at room temperature. TEM images of the nanoparticles were taken with Zeiss EM 900 instrument operated at an accelerating voltage of $120 \mathrm{kV}$ at room temperature. The samples for TEM measurements were prepared by placing a droplet of the colloidal solution onto a carbon-coated copper grid and allowing it to dry. Based on the TEM images, we determined the size distributions of the nano product by

Int. J. Radiat. Res., Vol. 18 No. 4, October 2020 
counting at least 300 particles. The concentrations of GNPs in $\mu \mathrm{g} / \mathrm{ml}$ were measured by the inductively coupled plasma optical emission spectrometry (ICP-OES). The surface charge was determined by a ZEN 3600 nanosizer (Malvern, UK). The hydrodynamic size of the GNPs was measured via Dynamic Light Scattering method (DLS).

\section{Cell culture}

4T1 tumour cells were purchased from the Pasteur Institute (Tehran, Iran).

ATCC Number for cell line used is CRL-2539. 4T1 is a breast cancer cell line derived from the mammary gland tissue of a mouse. 4T1 cells are epithelial and has several characteristics that make it a suitable experimental animal model for human mammary cancer.

We used the DMEM- high glucose culture medium to cultivate these cells with $10 \%$ FBS and $1 \%$ Penstrep. The cells were kept inside the incubator at $5 \% \mathrm{CO}_{2}$ and adequate humidity at $37^{\circ} \mathrm{C}$. When the cells filled the culture flask surface significantly, using with the trypsin-EDTA solution (10\%), the cells were detached from the flask surface and after counting, they were injected.

\section{Tumor models}

Six to eight weeks old female BALB/c mice with a weight of 20 to $22 \mathrm{~g}$ were purchased from the Pasteur Institute (Tehran, Iran). The mice were kept in the central laboratory of Isfahan University of Medical Sciences at $23^{\circ} \mathrm{C} \pm 2^{\circ} \mathrm{C}$ and $65 \%$ moisture. To create a tumour model, $1 \times 10^{6}$ 4T1 tumour cells per mouse were implanted subcutaneously in the right dorsal of the mice. Approximately 15 days after implantation, when tumour volume reaches about $100 \pm 20 \mathrm{~mm}^{3}$, the treatment protocols were performed on the tumours (14).

\section{Animals' anaesthesia}

Before US irradiation, the mice were anesthetized by intraperitoneal injection of ketamine Hydrochloride $(60 \mathrm{mg} / \mathrm{kg})$ and xylazine $2 \%(6 \mathrm{mg} / \mathrm{kg})^{(14)}$.

\section{US waves system}

Mice were exposed to ultrasound waves with a therapeutic ultrasound unit (215X, Novin, Iran) at a frequency of $1 \mathrm{MHz}$ with $0.5,1$ and 2 $\mathrm{W} / \mathrm{cm}^{2}$ intensities in a continuous mode for 2 minutes. Acoustic calibration was done in a degassed water tank using an US balance power meter (UPM 2000; Netech Co., Grand Rapids, MI). The uncertainty of calibration was less than $\pm 1 \mathrm{~mW}$. In this research, all intensities were spatial-temporal average.

\section{Treatment protocol}

The mice were divided into 4 groups (each containing 10 mice): (1) control, (2) GNPs, (3) ultrasound alone and (4) ultrasound in the presence of GNPs (US+ GNPs). For groups 2 and 4 , a single dose GNP was injected in the centre of tumours. The injected dose was $7 \mathrm{mg}$ of GNP per $\mathrm{kg}$ of mouse weight (15).

Before ultrasound irradiation, the tumour region was shaved with an animal shaver. The shaved region was then treated with depilatory cream; then, it was washed with liquid soap and rinsed. At that point, the skin was immersed in the water bath and the tumour centre was exposed to the ultrasound waves.

To avoid thermal effects due to ultrasound irradiation, cold degassed water was used. For the mice in the group treated by US+GNPs, the tumours were exposed to the ultrasound 24 hours after GNPs injection (1). The experimental set-up for the ultrasound exposure to the animal tumour models is shown in figure 1.

Ethical approval of Isfahan University of Medical Sciences with registration number: IR.MUI.REC.1396.1.138

Date of issue: $1396 / 10 / 19$

Research design code: 196138

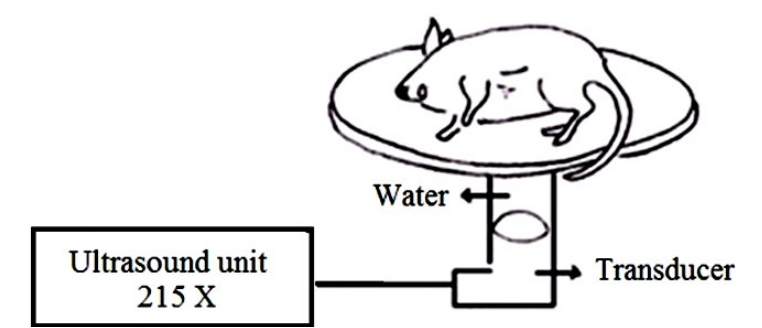

Figure1. The experimental set-up for ultrasound exposure to the animal tumour models. 


\section{Evaluation of treatment efficacy}

Treatment efficacy was evaluated via daily measurement of tumour diameters $(a, b)$ and tumour thickness (c). A digital caliper with 0.01 $\mathrm{mm}$ precision was used for the measurements; the tumour volume (V) was measured through the following equation: $V=\pi / 6(a \times b \times c)$

Tumours were measured up to 20 days after treatment. For each tumour, the treatment day was considered as a baseline (day 0) and the relative tumour volume (RTV) on later days was normalized accordingly.

According to daily variations of the RTV, $\mathrm{T}_{2}$ (time required for a tumour to reach twice its initial volume measured on the treatment day) and $\mathrm{T}_{5}$ (time required for a tumour to reach 5 times its initial volume measured on the treatment day) (1) of the tumours were calculated in each group.

\section{Statistical validation}

The obtained data were analysed using SPSS version 22 statistical software. The Mann-Whitney test was applied to compare RTVs with a confidence level of $95 \%$. The $\mathrm{T}_{2}$ and $\mathrm{T}_{5}$ of the tumours were compared in different groups using a one-way ANOVA test. $P<0.05$ was considered significant.

\section{RESULTS}

UV-Vis spectrometry was utilized to characterize the synthesized GNP (figure 2). Evidently, the GNP displays characteristic surface Plasmon absorption at $520 \mathrm{~nm}$, indicative of the formation of GNPs.

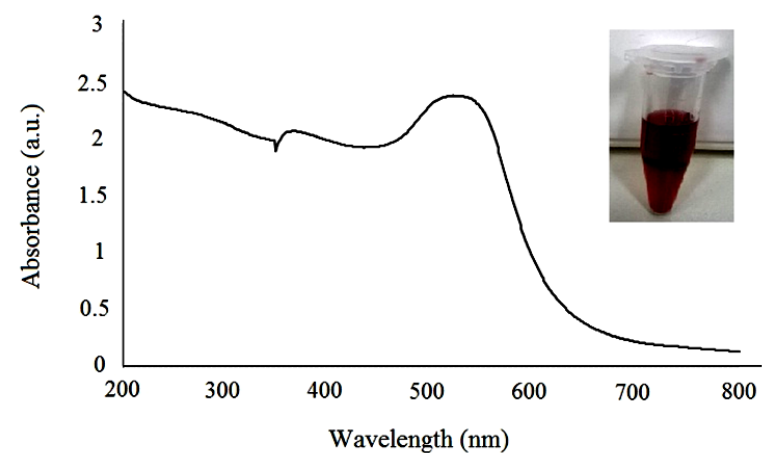

Figure 2. UV-Vis absorption spectra of GNPs.
The size distribution and morphology of the synthesized GNPs were characterized using TEM (figure 3). It can be noted that the synthesized GNPs have spherical shape around $23 \mathrm{~nm}$ in diameter.

The hydrodynamic sizes of the GNPs were measured via DLS to be $22.4 \mathrm{~nm}$ (figure 4). Zeta potential measurements were also employed to confirm the good reaction. The surface potential of the GNPs was revealed $-28.5 \mathrm{mV}$ (figure 4).

Measuring tumours growth, 7 and 14 days after treatment in the different groups, is shown in figure 5.
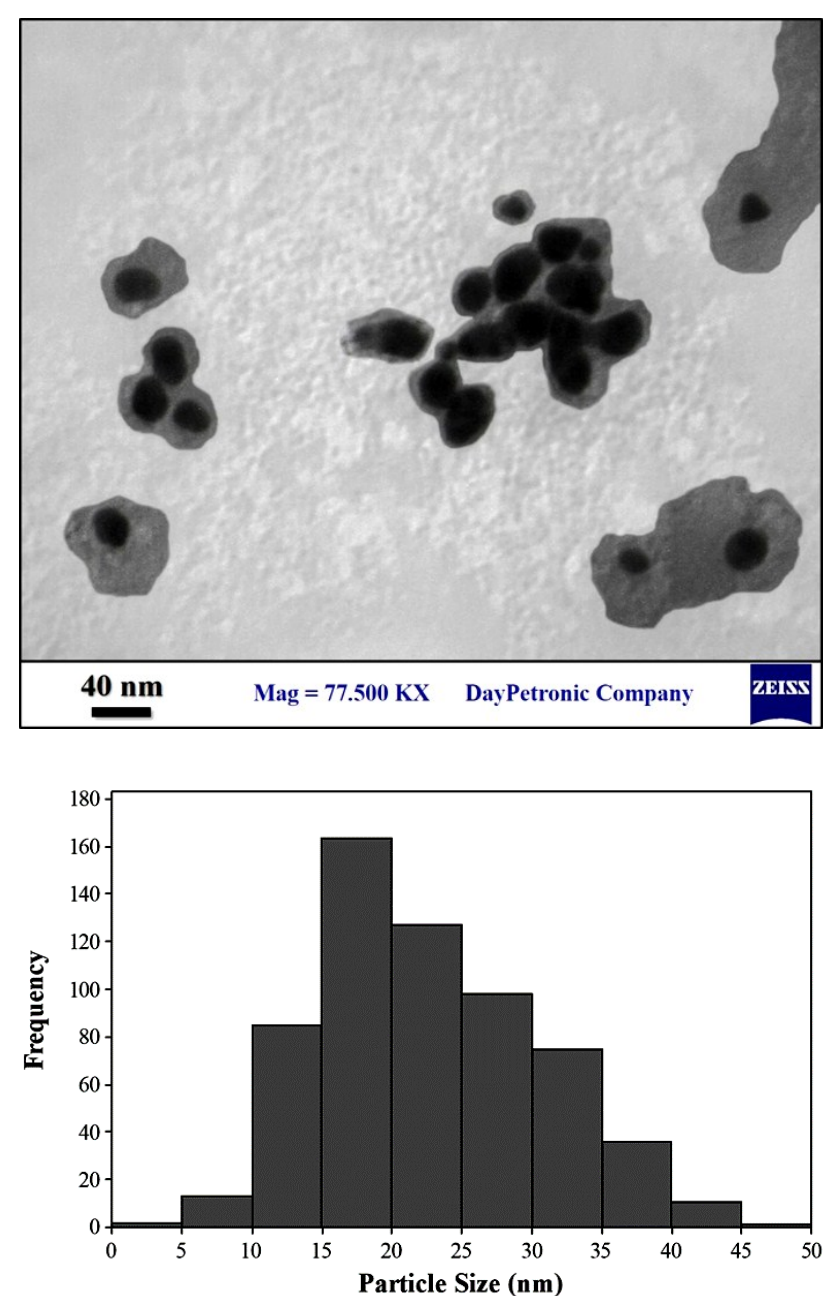

Figure 3. TEM images and size distributions of $\sim 23 \mathrm{~nm}$ GNPs.

Int. J. Radiat. Res., Vol. 18 No. 4, October 2020 


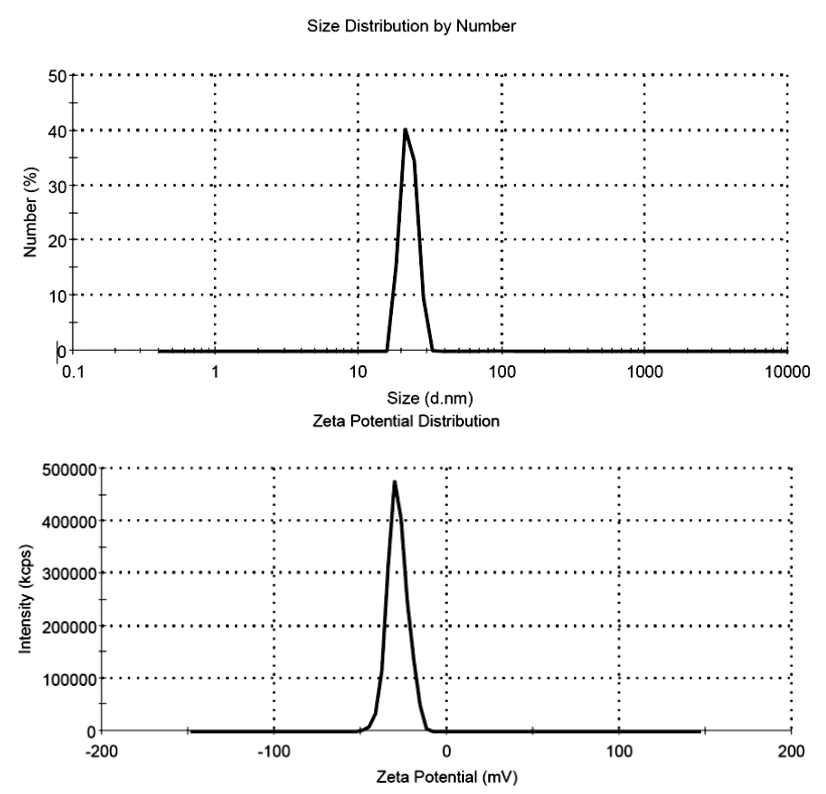

Figure 4. Dynamic light scattering measurements (DLS) of GNPs (peak: $22.4 \mathrm{~nm}$ ) and Zeta potential measurements of GNPs (peak: -28.5 mV).

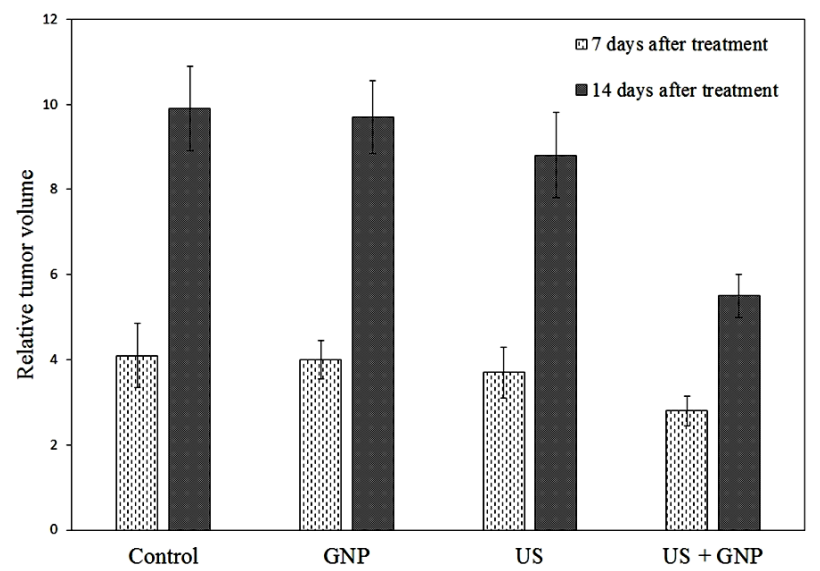

Figure 5. RTV 7 and 14 days after treatment in the different groups following $1 \mathrm{MHz}$ ultrasonic irradiation with $2 \mathrm{~W} / \mathrm{cm}^{2}$ intensity.

No therapeutic effect on tumours was seen in the control and GNPs groups. Further, there were no significant differences between the GNPs and control groups $(P=0.5)$. These results imply that GNPs had no detectable cytotoxicity.

Our results showed that ultrasound irradiation alone has no significant antitumour effects; however, its effects are enhanced by the ultrasound in the presence of GNPs (US+GNPs) in 7 days after treatment. In 14 days after treatment, the tumour inhibitory effect was significant when ultrasound irradiation alone and US+GNPs were applied.

In order to follow up the treatment, the tumor volumes in different groups were measured and compared with each other on each day after the treatment initiation.

Statistical comparison of the results revealed a significant decrease in the tumor volume in the US+GNPs group compared to those in the control and GNPs ones in 7 days after treatment $(P<$ 0.04). Furthermore, a significant difference between the US+GNPs and other groups was observed 14 days after treatment $(P<0.02)$. Our results also revealed that the tumour therapeutic effect became better as time passed after treatment.

Both the $\mathrm{T}_{2}$ and $\mathrm{T}_{5}$ can be good indicators for evaluating tumor growth. The $\mathrm{T}_{2}$ and $\mathrm{T}_{5}$ of tumours volume in treatment groups are shown in figure 6.

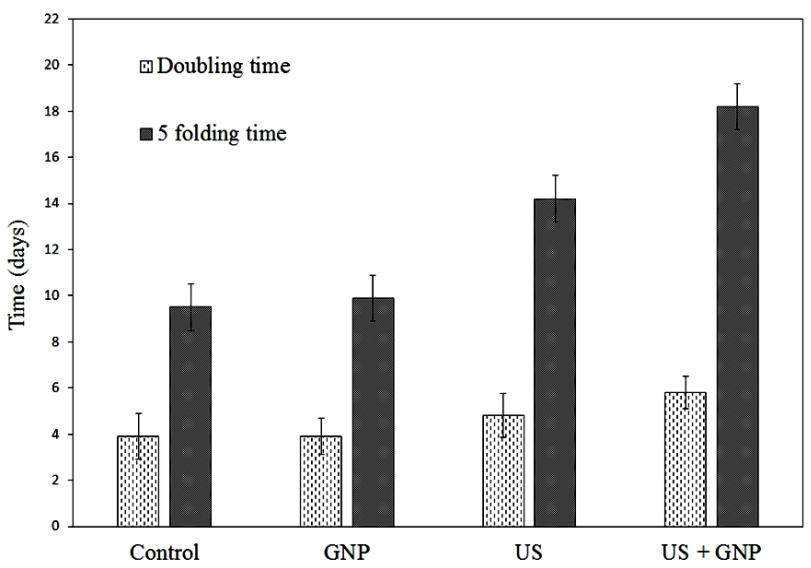

Figure 6. Average $T_{2}$ and $T_{5}$ in the treatment groups following $1 \mathrm{MHz}$ ultrasonic irradiation with $2 \mathrm{~W} / \mathrm{cm}^{2}$ intensity.

The $\mathrm{T}_{2}$ and $\mathrm{T}_{5}$ can represent tumour rapid and delayed responses to treatment, respectively.

The longest $T_{2}$ and $T_{5}$ were observed in the US+GNPs and ultrasound alone groups, respectively. The $\mathrm{T}_{2}$ was not significant among all groups $(P>0.07)$, whereas the $\mathrm{T}_{5}$ showed a significant difference between US+GNPs and the other groups $(P<0.01)$.

Figures 7 (a) and 7 (b) show RTV, 7 and 14 days after treatment in the field of $1 \mathrm{MHz}$ ultrasound waves at $0.5,1$, and $2 \mathrm{~W} / \mathrm{cm}^{2}$ intensities in the different groups respectively. 

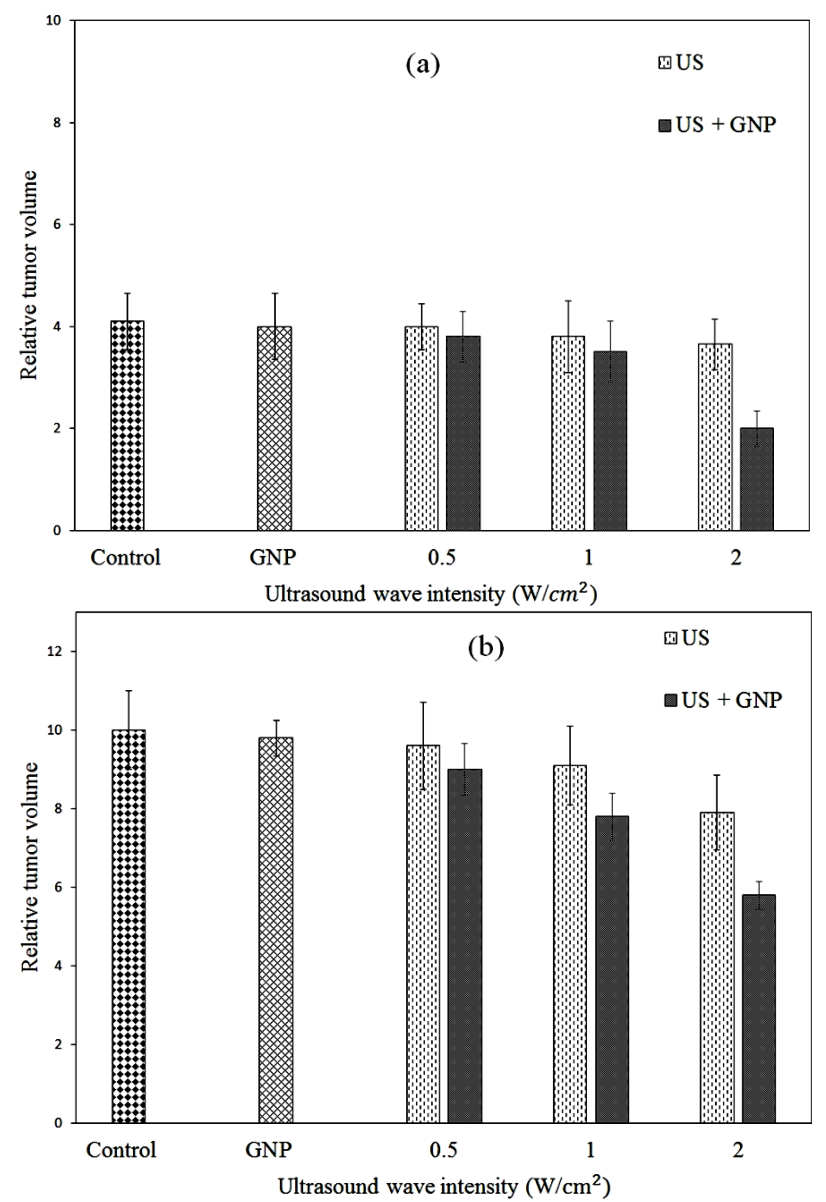

Figure 7. RTV, (a) 7 and (b) 14 days after treatment in the field of $1 \mathrm{MHz}$ ultrasound waves at $0.5,1$, and $2 \mathrm{~W} / \mathrm{cm} 2$ intensities.

Ultrasound irradiation alone showed a non-significant antitumour effect in the different intensities, which was enhanced by the administration of GNPs in different intensities. The inhibitory effect was significant when ultrasound in the presence of GNP was applied.

There was a significant difference in the RTV, 7 days after treatment between US+GNPs and ultrasound alone groups at $2 \mathrm{~W} / \mathrm{cm}^{2}$ intensity, $(\mathrm{P}<0.05)$; however, there is no significant difference in the RTV between US+GNPs and ultrasound alone groups at 0.5 and $1 \mathrm{~W} / \mathrm{cm}^{2}$ intensities $(\mathrm{P}>0.3)$.

Statistical comparison of the results showed a significant difference in the RTV, 14 days after treatment between the US+GNPs and ultrasound alone groups at 1 and $2 \mathrm{~W} / \mathrm{cm}^{2}$ intensities $(\mathrm{P}<$ 0.03); but the difference in the RTV between the US + GNPs and ultrasound alone groups was not significant at 0.5 intensity $(\mathrm{P}=0.1)$.

\section{DISCUSSION}

In the present study, gold nanoparticles have been obtained through a facile route by the chemical reduction of $\mathrm{HAuCl} 4$ in the presence of citrate. Furthermore, morphologies of the nano product was observed under transmission electron microscopy (TEM) operated at $120 \mathrm{kV}$. As is shown in Fig. 3, the nano sample with 23 $\mathrm{nm}$ in diameter has been achieved through using $350 \mu \mathrm{L}$ of $1 \%$ of trisodium citrate solution to 50 $\mathrm{mL}$ of $0.01 \% \mathrm{HAuCl} 4$ solution.

The main concern of researchers on increasing the therapeutic efficiency of ultrasound is to provide target tissue selectivity and to reduce the ultrasound intensity needed for the occurrence of inertial cavitation.

There are a few reports that suggest the nanoparticles existence in a liquid creates the nucleation sites for the cavitation during ultrasound exposure and a significant decrease of the acoustic cavitation onset threshold and increase in the number of bubbles $(9,10)$.

Chen et al. believe that cavitation increases in the presence of the microparticles due to increasing the number of cavities in the liquid (16).

The cavitation potential was investigated via sonoluminescence detection and terephthalic acid chemical dosimetry methods at therapeutic intensities of ultrasound (17). In that research, sonoluminescence was monitored on agar gel phantoms. When the agar gel phantom is exposed to ultrasound waves, cavitation may occur in the gel, and this leads to free radical production. This results in the production of sonoluminescence emission (17).

In this study, ultrasound waves were utilized at a frequency of $1 \mathrm{MHz}$ at $0.5,1$ and $2 \mathrm{~W} / \mathrm{cm}^{2}$ intensities in a continuous mode in the presence of GNPs $(23 \mathrm{~nm})$ as a way for increasing destructive effects on the tumours.

In the first stage of such investigations, there was a restriction related to GNPs distribution in tumours.

De Jong et al. reported that GNPs' Int. J. Radiat. Res., Vol. 18 No. 4, October 2020 
biodistribution is size-dependent (18). They showed that the nanoparticles, which are smaller than $10 \mathrm{~nm}$, are distributed widely in different organs including the heart, lungs, liver and also in the blood flow (18).

Other researchers revealed that, nanoparticles could be accumulated in some structures, such as the liver and spleen, for a long time post-intravenous injection regardless of their size, shape and dose.

In 2010, Balasubramanian et al. showed the rapid biodistribution of GNPs and also changes of gene expression in the liver and spleen in rats after intravenous injection (19).

In our study, GNPs were injected as intratumoural in order to get rid of this limitation.

Further, treatment efficacy was evaluated by different methods, including RTV, $\mathrm{T}_{2}$ and $\mathrm{T}_{5}$ of the tumours.

There were no significant differences between the GNPs and control groups. These results show that GNPs had no detectable cytotoxicity.

In 2018, the in vitro investigation performed by Shanei et al. approved the cancerous cells destruction using low level ultrasound in the presence of GNPs on 4T1 cells (20); GNPs showed no cytotoxicity.

So far, there have been many pieces of research concerning GNPs cell cytotoxicity in the living systems. However, there are two conflicting conclusions among the previous investigations. A small group of investigators insisted that GNPs are generally non-toxic while another group of scientists demonstrated the existence of toxicity in their researches (21-23). The emergence of various opinions is mainly due to the variations in related study factors such as GNPs' size, shape, surface charge, as well as coating material that can lead to different results in determining GNPs' interactions with biomolecules, cell lines and tissues.

In 2007, a study reported that the cytotoxicity of GNPs is dependent on the GNPs size (24). Smaller particle size (1-2 nm) was toxic, while larger sizes were nontoxic (24).

A significant difference between the US+GNPs and other groups was observed 14 days after treatment $(P<0.02)$, which confirms the effect of nucleating cavitation in the presence of GNPs and the role of GNPs in increasing efficiency of ultrasonic treatments.

Our research results showed that the therapeutic effect became better as time passed after treatment. Such results were also confirmed in other studies.

In 2013, an in vivo investigation showed the cavitation in the presence of GNPs as an approach for improving tumours therapeutic effects (25).

Their results showed that ultrasound irradiation alone has no significant antitumour effects, but its effects are enhanced by ultrasound waves in the presence of GNPs.

That study showed a significant difference in the RTV 15 days after treatment between the US+GNPs group and the other groups $(\mathrm{P}<0.04)$; however, there was no significant difference in the RTV 8 days after treatment between the US+GNPs and the other groups $(P>0.07)$.

In our research, the RTV of the US+GNPs group was significantly different in comparison with the other groups 7 days after treatment. This finding can be related to the larger size of GNPs or type of tumour cells. Size of GNPs in Sazgarnia et al. study was $7 \mathrm{~nm}$, and the study was on colon carcinoma tumour; whereas in our study, the size of GNPs was $23 \mathrm{~nm}$, and was conducted on a breast tumour model.

The effect of GNPs in different sizes on the cavitation activity have been investigated by detecting and quantifying free hydroxyl radicals in TA solutions containing GNPs in different sizes by using $1 \mathrm{MHz}$ low level ultrasound (26). In that research, TA solution was also utilized as a chemical dosimeter to quantify the free hydroxyl radicals generated by the collapse of the inertial cavities resulting from low-intensity ultrasound. This dosimetry is based on the fluorometric method, which is very sensitive to hydroxyl radical measurement.

In that study, it has been shown that the number of cavitation bubbles is increased with a rise in the size of GNPs, which results in an enhancement of the number of hydroxyl radicals 
(26).

In the present study, ultrasound exposure was performed by a planar transducer without any significant increase in media temperature, indicating that the therapeutic effects were not related to hyperthermia. Therefore, it was anticipated that cavitation might have acted during ultrasound irradiation.

Our results showed that the inhibitory effect became better as time passed after treatment. Such findings were also confirmed in other similar studies. Hachimine et all investigated sonodynamic cancer therapy using a novel porphyrin derivative. They showed that the growth of MKN-45 tumours was significantly inhibited by ultrasound + the porphyrin derivative 15 days after treatment in comparison with a control group (27).

The longest $T_{2}$ and $T_{5}$ were observed in the US+GNPs and ultrasound alone groups, respectively. The $T_{2}$ was not significant among all groups, whereas the $T_{5}$ showed a significant difference between US+GNPs and the other groups.

Since the $\mathrm{T}_{2}$ was not significant; whereas the $\mathrm{T}_{5}$ was, it seems that the antitumour effects of US+GNPs provide a delayed response. According to these results, the best response to the treatment was observed in the US+GNPs group.

Our results revealed that any increase in the intensity of the ultrasound waves would be associated with a decrease in the RTV. There was a significant difference in the RTV between US+GNPs and ultrasound alone groups at $2 \mathrm{~W} /$ $\mathrm{cm}^{2}$ intensity; however, no significant difference was observed in the RTV between US+GNPs and ultrasound groups at 0.5 and $1 \mathrm{~W} / \mathrm{cm}^{2}$ intensities.

In 2012, in a study, the amount of $\mathrm{OH}$ radicals' production versus ultrasound intensity has been investigated (9). Sazgarnia et al. reported that with increasing ultrasound intensity, the $\mathrm{OH}$ radical production was increased. The obtained results by ultrasound irradiation to the terephthalic acid solutions indicated that the parameters such ultrasound intensity are effective in $\mathrm{OH}$ radical production and, in turn, in the production of cavitation (9).

\section{CONCLUSION}

The main concern of this research on increasing the therapeutic efficiency of ultrasound is to provide target tissue selectivity and to reduce the ultrasound intensity, needed for the occurrence of inertial cavitation with minimal side effects to normal tissues. Combined effects of ultrasound and GNPs can be used as a method for increasing the therapeutic efficiency on tumor cells.

\section{ACKNOWLEDGEMENT}

This work was performed in Isfahan University of Medical Sciences. The authors would like to thank the staff of the central laboratory of Isfahan University of Medical Sciences that have kindly cooperated in this research.

Conflicts of interest: Declared none.

\section{REFERENCES}

1. Liu Q, Wang X, Wang $P$, Xiao L (2007) Sonodynamic antitumour effect of protoporphyrin IX disodium salt on S180 solid tumour. Chemotherapy, 53(6): 429-436.

2. Kennedy JE (2005) High-intensity focused ultrasound in the treatment of solid tumours. Nat Rev Cancer, 5(4): 321327.

3. Ivone M, Pappalettere C, Watanabe A, Tachibana K (2016) Study of cellular response induced by low intensity ultrasound frequency sweep pattern on myelomonocytic lymphoma U937 cells. J Ultrasound, 19(3): 167-174.

4. Yu T, Wang Z, Mason TJ (2004) A review of research into the uses of low level ultrasound in cancer therapy. Ultrason Sonochem, 11(2): 95-103.

5. Frenkel V (2008) Ultrasound mediated delivery of drugs and genes to solid tumours. Adv Drug Deliv Rev, 60 (10): 1193-1208.

6. Zupanc M, Pandur Z, Stepišnik Perdiha T, Stopar D, Petkovšek M, Dular M (2019) Effects of cavitation on different microorganisms: The current understanding of the mechanisms taking place behind the phenomenon. A review and proposals for further research. Ultrasonics Sonochemistry, 57: 147-165.

7. Shanei A and Sazgarnia A (2019) An overview of therapeutic applications of ultrasound based on synergetic effects

Int. J. Radiat. Res., Vol. 18 No. 4, October 2020 
with gold nanoparticles and laser excitation. Iran J Basic Med Sci, 22(8): 848-855.

8. Ashokkumar M (2011) The characterization of acoustic cavitation bubbles - An overview. Ultrason sonochem, 18 (4): 864-872.

9. Sazgarnia A and Shanei A (2012) Evaluation of Acoustic Cavitation in Terephthalic Acid Solutions Containing Gold Nanoparticles by the Spectrofluorometry Method. Int J Photoenergy, 10: 1-5.

10. Tuziuti T, Yasui K, Sivakumar M, Lida Y, Miyoshi N (2005) Correlation between Acoustic Cavitation Noise and Yield Enhancement of Sonochemical Reaction by Particle Addition. J Phys Chem A, 109(21): 4869-4872.

11. Huang X, Jain PK, El-Sayed IH, El-Sayed MA (2008) Plasmonic photothermal therapy (PPTT) using gold nanoparticles. Lasers Med Sci, 23(3): 217-228.

12. Paciotti GF, Myer L, Weinreich D, Goia D, Pavel N, McLaughlin RE, Tamarkin L (2004) Colloidal Gold: A Novel Nanoparticle Vector for Tumour Directed Drug Delivery. Drug Deliv, 11(3):169-183.

13. Grabar KC, Freeman RG, Hommer MB, Natan MJ (1995) Preparation and characterization of $\mathrm{Au}$ colloid monolayers. Anal Chem, 67(4): 735-743.

14. Kim K-H, Kim J-K, Lee D-H (2011) Sonodynamic induced antitumour effect of radachlorin on solid tumour. Curr Appl Phys, 11(3): 559-563.

15. Sazgarnia A, Shane A, Taheri AR, Tayyebi N, Eshghi $H$, Attaran N, Shanei MM (2013) Therapeutic effects of acoustic cavitation in the presence of gold nanoparticles on a colon tumour model. J Ultrasound Med, 32(3): 475-483.

16. Chen H, Wang J, Chen D (2009) Cavitation damages on solid surfaces in suspensions containing spherical and irregular microparticles. Wear, 266(1): 345-348.

17. Sazgarnia A, Shanei A, Eshghi H, Hassanzadeh-Khayyat M, Esmaily H, Shanei MM (2013) Detection of sonoluminescence signals in a gel phantom in the presence of Protoporphyrin IX conjugated to gold nanoparticles. Ultrasonics, 53(1): 29-35.

18. De Jong WH, Hagens WI, Krystek $P$, Burger MC, Sips AJ,
Geertsma RE (2008) Particle size-dependent organ distribution of gold nanoparticles after intravenous administration. Biomaterials, 29(12):1912-19.

19. Balasubramanian SK, Jittiwat J, Manikandan J, Ong CN, Yu LE, Ong WY (2010) Biodistribution of gold nanoparticles and gene expression changes in the liver and spleen after intravenous administration in rats. Biomaterials, 31(8): 2034-2042.

20. Shanei A, Attaran N, Mirzaeiyan M, Salamat MR, Hejazi H (2018) Cancerous cells destruction using low level ultrasound in the presence of gold nanoparticles: in vitro study on 4T1 cells. Current Nanoscience, 14(4): 329-334.

21. Patra HK, Banerjee S, Chaudhuri U, Lahiri P, Dasgupta AK (2007) Cell selective response to gold nanoparticles. Nanomedicine, 3(2): 111-119.

22. Zhang XD, Guo ML, Wu HY, Sun YM, Ding UQ, Feng $X$, Zhang LA (2009) Irradiation stability and cytotoxicity of gold nanoparticles for radiotherapy. Int I Nanomedicine, 4: $165-173$.

23. Peng G, Tisch U, Adams O, Hakim M, Shehada N, Broza YY, Billan S, Abdah-Bortnyak R, Kuten A, Haick H (2009) Diagnosing lung cancer in exhaled breath using gold nanoparticles. Nat Nanotechnol, 4(10): 669-673.

24. Pan $Y$, Neuss $S$, Leifert A, Fischler $M$, Wen F, Simon $U$, Schmid G, Brandau W, Jahnen-Dechent W (2007) Sizedependent cytotoxicity of gold nanoparticles. Small, 3(11): 1941-1949.

25. Sazgarnia A, Shanei A, Taheri AR, Tayyebi Meibodi N, Eshghi H, Attaran N, Shanei MM (2013) Therapeutic Effects of Acoustic Cavitationin the Presence of Gold Nanoparticles on a Colon Tumour Model. J Ultrasound Med, 32(3): 475-483.

26. Shanei A and Shanei MM (2017) Effect of gold nanoparticle size on acoustic cavitation using chemical dosimetry method. Ultrason sonochem, 34: 45-50.

27. Hachimine K, Shibaguchi H, Kuroki M, Yamada H, Kinugasa $T$ (2007) Sonodynamic therapy of cancer using a novel porphyrin derivative, DCPH-P-Na(I), which is devoid of photosensitivity. Cancer Sci, 98(6): 916-920. 
